\title{
LA FAUNA FLEBOTOMICA (DIPTERA: PSYCHODIDAE) DE TRES PROVINCIAS DE LA REGION NOR ORIENTAL DEL MARAÑON, PERU
}

\author{
Abraham G. Cáceres, Eunice A. Bianchi Galati, \\ François Le Pont y César Velásquez
}

\begin{abstract}
En localidades ubicadas entre los distritos de San José de Lourdes $105^{\circ} 03^{\prime}$ Longitud Sur), provincia de San Ignacio y Lonya Grande (06 $05^{\prime} 30^{\prime \prime}$ Longitud Sur), provincia de Utcubamba, Región Nor Oriental del Marañón, donde la bartonellosis bumana o Verruga Peruana es endémica, se capturaron 2774 flebótomos entre 1987 y 1992; identificándose 13 especies; 11 corresponden al género Lutzomyia, 1 a Brumptomyia $y$ 1 a Warileya. De las especies capturadas, Lutzomyia sp. similar a Lutzomyia serrana $y$ Lutzomyia maranonensis sp. n., Galati, Cäceres $\varepsilon$ Le Pont, son las mas abundantes (89.74\%), por lo que, se especula que pueden ser consideradas vectores potenciales de la Verruga Peruana. Se reporta Lutzomyia verrucarum (Townsend, 1913) por primera vez para la provincia de Utcubamba.
\end{abstract}

Palabras-claves: Pblebotominae. Perú. Lutzomyia, Brumptomyia, Warileya, bartonellosis. Verruga Peruana. Enfermedad de Carrión.

La bartonellosis humana, conocida como Verruga Peruana o Enfermedad de Carrión está predominantemente localizada en el Perú, siendo endémica en los valles occidentales, interandinos y orientales del Perú ${ }^{14}{ }_{22}{ }^{23} 26$; asimismo, está presente en Ecuador ${ }^{8} 1520$ y Colombia ${ }^{24}$.

Las provincias de Jaén, San Ignacio y Utcubamba están ubicadas en valles interandinos del norte del Perú. La primera es considerada zona verrucógena desde hace aproximadamente nueve décadas ${ }^{23}{ }^{26}$, ya que, en ciertas localidades la Verruga Peruana es endémica, presentándose cada año numerosos casos humanos. La provincia de San Ignacio es considerada zona verrucógena desde hace cinco décadas ${ }^{98}$ y los casos se presentan esporádicamente; esto mismo sucede en la provincia de Utcubamba. Las provincias mencionadas pertenecen a la Región Nor Oriental del Maranón (RENOM).

No se tiene información precisa referente a la existencia del vector(es) de la Verruga

Instituto Nacional de Salud, Lima Perú; Departamento de Epidemiología, Faculdade de Saúde Pública, Universidade de São Paulo, São Paulo, SP Brasil; ORSTOM, París, Francia; Hospital General de Bagua, Región Nor Oriental del Marañón, Perú.

Endereço para correspondência: Dr. Abraham G. Cáceres. Instituto Nacional de Salud, Ap. 451, Lima 100, Perú. Recebido para publicação em 27/09/94.
Peruana en Jaén, San Ignacio y Utcubamba ${ }^{911}$. La necesidad de aclarar esta información es el motivo, por el cual, a partir de 1987, se iniciaron estudios entomológicos para: 1) verificar la existencia de $L$. verrucarum (Townsend, 1913), considerada el vector principal de esta enfermedad; 2) conocer la fauna flebotómica existente en las tres provincias arriba mencionadas, donde la bartonellosis humana constituye un problema de Salud Pública.

\section{MATERIAL Y METODOS}

Area de estudio. Los flebótomos fueron capturados en valles interandinos del norte del Perú, en localidades ubicadas entre $5^{\circ} 3^{\prime} \mathrm{LS}$ 78 $53^{\prime} \mathrm{LO}$ y 605'30"LS - 78'30'29"LO de la RENOM.

Dichas localidades pertenecen a los distritos de San José de Lourdes y La Coipa (provincia de San Ignacio) ubicadas en el valle Chinchipe; a Jaén y Santa Rosa (provincia de Jaén) situada en el valle Chamaya y Chinchipe; y a los distritos de El Milagro y Lonya Grande (provincia de Utcubamba) ubicadas en la margen derecha del Río Marañón (Figura 1).

Capturas de Flebótomos. En cuatro períodos distintos entre 1987 y 1992 se realizaron capturas de flebótomos:

Noviembre, 1987

- Provincia de San Ignacio: En los distritos San José de Lourdes y El Milagro; en ambas 
Cáceres $A G$, Galati $E A B$, Le Pont $F$, Velásquez C. La fauna flebotomica (Diptera: psychodidae) de tres provincias de la Región Nor Oriental del Marañon, Perú. Revista da Sociedade Brasileira de Medicina Tropical 28:215-221, jul-set, 1995.

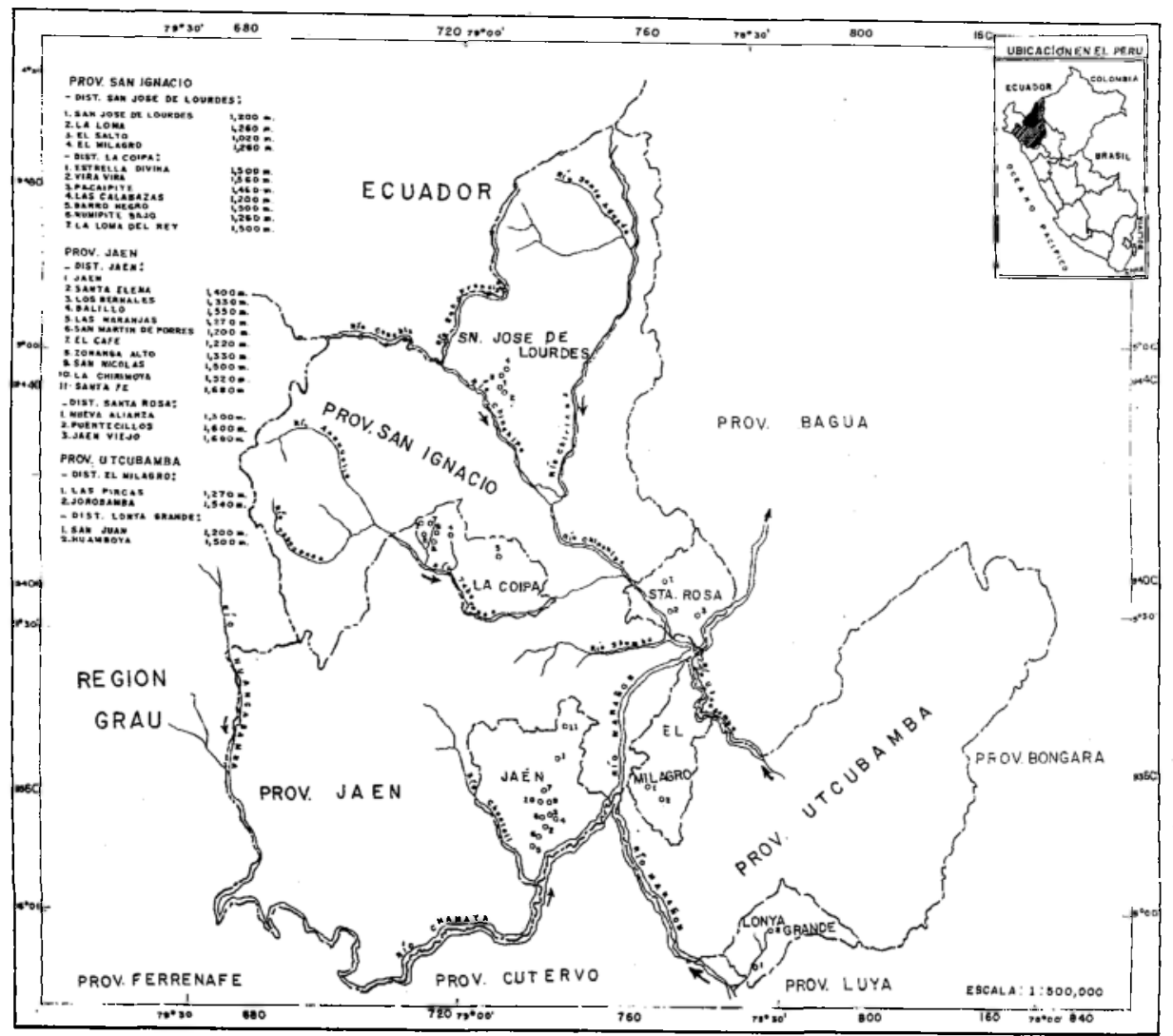

Figura 1- Localidades donde se han capturado flebótomos entre 1987 y 1992; Región Nor Ortental del Maranón, Peri

localidades los flebótomos fueron capturados en grietas, cuevas naturales y/o artificiales y en raíces de plantas de café, cón aspirador manual, linterna de mano y humo de cigarrillo.

\section{Setiembre, 1988}

- Provincia de San Ignacio: Distrito de San José de Lourdes, El Milagro: a) campo abierto, se capturó con aspirador manual, linterna de mano y humo de cigarrillo; b) cebo humano desde las 15.00 hasta las 17.00 horas.

\section{Marzo, 1992}

- Provincia de San Ignacio: Distrito de San José de Lourdes: en localidades del El Milagro y La Loma, las capturas fueron en dormitorios con trampas de luz tipo CDC, 17.00-6.00 horas; en El Salto la captura fué con cebo humano desde 18.00 hasta 20.30 horas.

Distrito de La Coipa: en localidades de Estrella Divina, Vira Vira, Pacaipite, Las
Calabazas y Barro Negro las capturas fueron en dormitorios con trampas de luz tipo CDC de 17.00 a 6.00 horas; en Pacaipite y Rumipite Bajo fué con trampa Shannon desde 19.00 hasta 22.00 horas; en Loma del Rey se capturó en los dormitorios con aspirador manual, linterna de mano y humo de cigarrillo de 8.30 a 9.30 horas.

- Provincia de Jaén: Distrito de Jaén: en las localidades de Santa Elena se capturó en dormitorios con trampas de luz tipo CDC desde 17.00 hasta 6.00 horas.

Setiembre y Octubre, 1992

- Provincia de Jaén: Distrito de Jaén: en las localidades de Balillo, Los Bernales, San Martín de Porres, San Nicolas, Santa Fé y Zonanga Alto se capturaron en los dormitorios con trampas de luz tipo CDC de 17.00 a 22.00 horas; en Santa Fé fué con trampa Shannon entre 19.00 y 20.00 horas; en Balillo la captura fué con cebo humano desde 
Cáceres $A G$, Galati $E A B$, Le Pont $F$, Velásquez C. La fauna flebotomica (Diptera: psychodidae) de tres provincias de la Región Nor Oriental del Marañon, Peri. Revista da Sociedade Brasileira de Medicina Tropical 28:215-221, jul-set, 1995.

20.00 hasta 21.00 horas; en Zonanga Alto se capturó con cebo humano entre 19.30 y 21.00 horas; en La Chirimoya (San Nicolas) las capturas se realizaron en dormitorios empleando aspirador manual, linterna de mano y humo de cigarrillo, $19.00-20.00$ horas.

Distrito de Santa Rosa: en las localidades de Nueva Alianza, Puentecillos, Jaén Viejo las capturas fueron en dormitorios con trampas de luz tipo CDC, 17.00-22.00 horas; mientras que en Nueva Alianza fué con cebo humano, 19.00-21.00 horas.

Provincia de Utcubamba: Distrito de E] Milagro: en las localidades de Jorobamba y Las Pircas los flebótomos se capturaron en dormitorios con trampas de luz tipo CDC, 17.00-22.00 horas.

Distrito de Lonya Grande: en localidades de Huamboya y San Juan las capturas fueron en dormitorios con trampas de luz tipo CDC, 17.00-22.00 horas; mientras que en Huamboya se capturó con trampa Shannon, 18.00-22.00 horas.

Una sintesis del número de capturas y horas consta en la Tabla 1.

Los flebótomos fueron transferidos a viales de vidrio conteniendo alcohol $70 \%$, otros se conservaron en cajitas con sílica gel para mantenerlos en seco y posteriormente ser identificados.

Tabla 1 - Número de capturas y de boras por método de captura en distritos de la provincia de San Ignacio Jaén y Utcubamba, Región Nor Oriental del marañnon, Perú (1987-1992).

\begin{tabular}{|c|c|c|c|c|c|c|c|c|}
\hline \multirow[b]{3}{*}{ Provincia } & \multirow[b]{3}{*}{ Distritos } & \multicolumn{2}{|c|}{ CDC } & \multirow{2}{*}{\multicolumn{2}{|c|}{ Cebo humano }} & \multirow{3}{*}{$\begin{array}{c}\text { Trampa } \\
\begin{array}{c}N^{\circ} \text { de } \\
\text { capturas }\end{array}\end{array}$} & \multirow{3}{*}{$\begin{array}{c}\text { Shannon } \\
\begin{array}{l}\mathrm{N}^{\alpha} \mathrm{de} \\
\text { horas }\end{array}\end{array}$} & \multirow{3}{*}{ 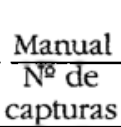 } \\
\hline & & $17-6 \mathrm{~h}$ & $17-22 \mathrm{~h}$. & & & & & \\
\hline & & $\begin{array}{l}\mathrm{N}^{Q} \mathrm{de} \\
\text { capturas }\end{array}$ & $\begin{array}{l}\text { No de } \\
\text { capturas }\end{array}$ & $\begin{array}{l}\mathrm{N}^{\circ} \text { de } \\
\text { capturas }\end{array}$ & $\begin{array}{l}N^{\circ} \text { de } \\
\text { horas }\end{array}$ & & & \\
\hline San Ignacio & S.J. de Lourdes & 2 & - & 2 & 4,0 & - & - & 2 \\
\hline San Ignacio & La Coipa & 5 & - & - & - & 2 & 7,0 & 1 \\
\hline Jaén & Jaén & 1 & 12 & 2 & 1,5 & 1 & 1,0 & 1 \\
\hline Jaén & Santa Rosa & - & 6 & 1 & 2,0 & 1 & 2,0 & - \\
\hline Utcubamba & Lonya Grande & - & 2 & - & - & 2 & 4,0 & - \\
\hline Utcubamba & El Milagro & - & 2 & - & - & - & - & - \\
\hline \multicolumn{2}{|c|}{ Total Node capturas } & 8 & 22 & 5 & & 6 & & 4 \\
\hline \multicolumn{2}{|c|}{ Total No de horas } & 104,0 & 110,0 & & 7,5 & & 14,0 & \\
\hline
\end{tabular}

\section{RESULTADOS}

Se capturaron 2,774 flebótomos, identificandose 11 especies de Lutzomyia, 1 de Warileya y 1 de Brimptomyia (Tablas 2 y 3); de ellas Lutzomyia sp. similar a $L$. serrana y $L$. maranonensis 'sp. $n$., Galati, Cáceres \& le Pont, destacan por su mayor densidad en las 3 provincias estudiadas.

Lutzomyia sp. similar a $L$. serrana fué la especie más frecuente de los flebótomos capturados con trampas tipo CDC (intradomicilios), mientras que, $L$. maranonensis sp. $n$., fué la que representó la mayor media horaria en captura con cebo humano en el peridomicilio, incluyendo machos (Tabla 3).

Lutzomyia sp. similar a $L$. serrana corresponde a la serie Serrana, y $L$. maranonensis sp. n., Galati, Cáceres \& Le Pont a la serie Evansi, mientras que, $L$. cajamarcensis sp. n., Galati, Cáceres \& Le Pont, L. verrucarum (Townsend, 1913), asî como, Lutzomyia sp. pertenecen a la serie Verrucarum. Estas 6 especies están incluidas en el subgénero Pifanomyia Ortiz \& Scorza, según Galati et al. (trabajo en prensa).

De los otros flebótomos capturados con cebo humano $L$. ayacucbensis, Cáceres \& Galati, 1988 representa la mayor frecuencia, seguido por $L$. castanea sp. $n$. y $L$. palliditborax $s p$. n., Galati \& Cáceres ${ }^{17}$, todos pertenecen al subgénero Helcocyrtomyia Barretto.

Lutzomyia sallesi Galvao \& Cautinho, 1939 presenta baja densidad con trampa tipo CDC y en capturas con cebo humano, pero fué la que más se capturó con aspirador manual. Su número fué superado solamente por Lutzomyia sp. similar a $L$. serrana, si se toma en cuenta ambos sexos.

Se señala por primera vez $L$. verrucarum y Warileya phlebotomanica para las provincias de Utcubamba y Jaén respectivamente. 
Cáceres $A G$, Galait E4B, Le Pont $F$, ielásquez C. La fauna flebotomica (Diptera: psychodidae) de tres provincias de la Región Nor Oriental del Marañon, Perú. Revista da Sociedade Brasileira de Medicina Tropical 28:215-221, jul-set, 1995.

Tabela 2 - Fauna flebotónica de tres provincias de la Región Nor Oriental del Marañon, Perú (1987-1992).

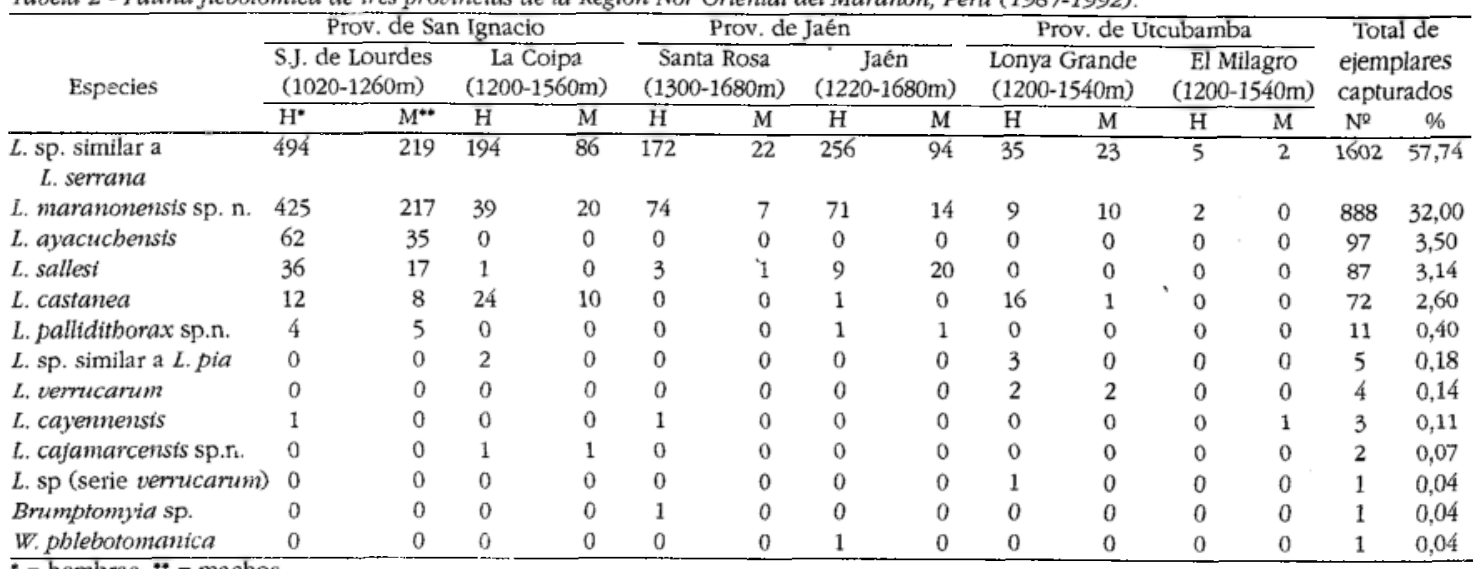

Tabela 3 -Mediu horar ia de las capturas de flebotominos realizadas com trampa CDC, cebo bumano, trampa de Shannon y media por captura manual en los distritos de las provincias de San Ignacio, Jaén y Utcubamba, Región Nor Oriental del marañon, Perü (1987-1992).

\begin{tabular}{|c|c|c|c|c|c|c|c|c|}
\hline \multirow[t]{2}{*}{ Especies } & \multicolumn{2}{|c|}{$\frac{\mathrm{CDC}}{\text { media horaria }}$} & \multicolumn{2}{|c|}{$\begin{array}{l}\text { Cebo humano } \\
\text { media horaria }\end{array}$} & \multicolumn{2}{|c|}{$\frac{\text { Trampa Shannon }}{\text { media horaria }}$} & \multicolumn{2}{|c|}{ Manual } \\
\hline & $\mathrm{H}^{*}$ & $M^{* *}$ & $\mathrm{H}$ & M & $\mathrm{H}$ & M & $\mathrm{H}$ & $M$ \\
\hline L. sp. similar a L. serrana & 5,28 & $1,9 \overline{7}$ & 5,20 & 2,00 & 1,07 & 0 & 4,25 & 6,50 \\
\hline L. maranonensis sp. $\mathrm{n}$. & 2,41 & 0,61 & 15,20 & 17,73 & 0,36 & 0,07 & 0,25 & 0,25 \\
\hline L. ayacucbensis & 0,13 & 0,12 & 4,13 & 1,47 & 0 & 0 & 1,25 & 0 \\
\hline L. sallesi & 0,09 & 0,15 & 0,40 & 0,13 & 0 & 0 & 6,75 & 1,75 \\
\hline L. castanea & 0,21 & 0,06 & 0,80 & 0,67 & 0,14 & 0 & 0 & 0 \\
\hline L. pallidithorax sp.n. & 0,01 & 0,01 & 0,40 & 0,67 & 0 & 0 & 0,25 & 0 \\
\hline L. sp. similar a L. pia & 0,03 & 0 & 0 & 0 & 0,14 & 0 & 0 & 0 \\
\hline L. verrucarum & 0,01 & 0,01 & 0 & 0 & 0 & 0 & 0 & 0 \\
\hline L. cayennensis & 0,02 & 0,02 & 0,13 & 0 & 0 & 0 & 0 & 0 \\
\hline L. cajamarcensis sp.n. & 0,01 & 0,01 & 0 & 0 & 0 & 0 & 0 & 0 \\
\hline L. sp (serie verrucarum) & 0,01 & 0 & 0 & 0 & 0 & 0 & 0 & 0 \\
\hline Brumptomyia sp. & 0,01 & 0 & 0 & 0 & 0 & 0 & 0 & 0 \\
\hline Warileya pblebotomanica & 0 & 0 & 0 & 0 & 0,07 & 0 & 0 & 0 \\
\hline
\end{tabular}

\section{DISCUSION}

La mayor densidad de Lutzomyia sp. similar a $L$. serrana y de $L$. maranoneissis $s p$. $n$. en intradomicilio $y$ por las capturas con cebo humano (Tabla 3), nos sugiere que estas especies pueden estar desempeñando el papel de vector (es) de la bartonellosis humana en la zona de estudio. Estas especies, están presentes en la provincia de ZamoraChinchipe (Ecuador), zona donde la leishmaniasis tegumentaria y la Verruga Peruana son endémicas ${ }^{18}$. Estas especies son mencionadas por Alexander y colaboradores como $L$ serrana y $L$. nevesi para Ecuador ${ }^{1}$.

Gamarra', hace referencia a que flebótomos colectados en 1941-42, en localidades de la provincia de San Ignacio fueron identificados como L. verrucarum. Asimismo, Herrer ${ }^{11}$ menciona que flebótomos procedentes de una localidad $(1500 \mathrm{~m})$ alrededor del río Tamborapa (Jaén) fueron identificados como L. verrucarum. Estos ejemplares no se encuentran en colecciones de Instituciones Públicas o privadas, es por eso, que no ha sido posible ser observado para confirmar la identificaçión. De nuestros datos, suponemos que dichos ejemplares corresponden a Lutzomyia sp. similar a $L$. serrana, ya que, ésta especie presenta algunas características morfológicas similares a $L$. verrucarum; además, que está presente en mayor densidad en las tres provincias estudiadas. 
Cáceres $A G$, Galati $E A B$, Le Pont $F$, Velásquez C. La fauna flebotomica (Diptera: psychodidae) de tres provincias de la Region Nor Oriental del Marañon, Perí. Revista da Sociedade Brasileira de Medicina Tropical 28:215-221, jul-set, 1995.

Lutzomyia verrucarum, vector natural de la Verruga Peruana o enfermedad de Carrión ${ }^{21621}$ 273031 está presente en los valles andinos occidentales desde $5^{\circ}$ hasta $13^{\circ} 25^{\prime}$ Latitud Sur asimismo, ha sido capturado en valles interandinos del Santa, Huallaga, Cajamarca, Cajabamba y Huancabamba ${ }^{410121319}$. En la zona de estudio, L. verrucarum, está presente en los alrededores del distrito de Lonya Grande (Utcubamba) margen derecha del Rio Marañón.

Herrer $^{1012}$ menciona que $L$. verrucarum fué capturado en Cajamarca, Celendín y Cajabamba, localidades ubicadas en la margen izquierda del Río Marañón.

Lutzomyia ayacuchensis fué descrita en el Perú a partir de especimenes procedentes de Parinacochas y Lucanas (Región Los Libertadores Wari), donde es el vector de la leishmaniasis cutánea andina ó "uta". También, está presente en el valle occidental e interandino de Huancabamba de la Región $\mathrm{Grau}^{5}$. En Ecuador, fué encontrada infectada en forma natural con Leishmania mexicana en focos de leishmaniasis tegumentaria ${ }^{29}$. Lutzomyia ayacuchensis, L. castanea sp. $n$. y $L$. palliditborax $s p$. $n$. pertenecen al subgénero Helcocyrtomyia Barretto, de los cuales en el Perú, algunas especies están implicadas en la transmisión de la leishmaniasis cutánea andina ó "uta" 725 . Lutzomyia castanea, también está presente en el valle occidental de Huancabamba, Región Grau (P Villaseca: comunicación personal, 1993); asimismo, se ha encontrado en Ecuador ${ }^{18}$ y Bolivia ( $F$ Le Pont: comunicación personal, 1994).

Lutzomyia sp. similar a $L$. pia colectada en La Coipa (San Ignacio) y Lonya Grande (Utcubamba), puede corresponder a la hembra de $L$. reclusa Fernadez \& Rogers, 1991; ya que $L$. reclusa descrita solamente por machos, es similar a $L$. pia y fué colectada en la provincia de Santa Cruz, en la misma Región donde se desarrolló nuestro estudio. Para confirmar esta hipótesis, se requiere de mayor número de ejemplares tanto machos como hembras de Lutzomyia sp. similar a L. pia y de L. reclusa.

Se señala por primera vez la presencia de L. sallesi para Jaén y San Ignacio; esta especie está presente, también en Huánuco ${ }^{3}$, Región Andrés A. Cáceres y, en el valle occidental de Huancabamba, Región Grau (P Villaseca: comunicación personal, 1993).
Lutzomyia cayennensis Floch \& Abonnenc fué señalada con reservas para el Perú por Martins et al., 1976. Esta especie está presente en las tres provincias estudiadas.

\section{Conclusiones}

Se señala, para las provincias de San Ignacio, Jaén y Utcubamba, RENOM la presencia de 13 especies de flebotominos: 1 de Brumptomyia, 1 de Warileya y 11 de Lutzomyia; entre éstas, cinco son especies nuevas: L. (Helcocyrtomyia) castanea, L. (Hel.) pallidithorax, L. (Pifanomyia) maranonensis, $L$. (Pif.) cajamarcensis, $L$. (Pif.) sp. similar a $L$. serrana y probablememnte la hembra de $L$. reclusa.

Las dos especies más frecuentes en el área de estudio fueron: Lutzomyia sp. similar a $L$. serrana y L. maranonensis.

En intradomicilio (capturas con trampas tipo CDC), se señala que la densidad de hembras de Lutzomyia sp. similar a $L$. serrana, fué casi 2 veces mayor en relación a $L$. maranonensis.

En peridomicilio (capturas con cebo humano), las hembras de L. maranonensis presentaron una densidad de casi 3 veces mayor que Lutzomyia sp. similar $L$. serrana. En las capturas con cebo humano, también apareció en proporción destacada $L$. ayacucbensis.

De las informaciones obtenidas, se puede sugerir que probablemente Lutzomyia sp. similar a $L$. serrana y $L$. maranonensis sean los principales vectores de la Verruga Peruana en las tres provincias estudiadas.

\section{SUMMARY}

Two thousand seven bundred and seventy four sand flies were captured since 1987 to 1992 in endemic areas of buman bartonellosis and Peruvian Verruga (district of San José de Lourdes, proutnce of San Ignacio - 05.03' LS, and district of Lonya Grande, province of Utcubamba - 06.05'30" $L S$ ) belonging to the Northeast region of Maranion, Peru. Thirteen species were identified, 11 belonging to the genus Lutzomyia, 1 to Brumptomyia and the other to Warileya. Lutzomyia sp, close to L. serrana and $\mathrm{L}$. maranonensis n. sp., Galati, Cáceres \& Le Pont are more prevalent ( $89.74 \%$ of the individuals captured). Suggests working of the kind. Lutzomyia verrucarum (Townsend, 1913) is reported in Utcubamba province for the first time. 
Cáceres $A G$, Galati $E A B$, Le Pont $F$, Velásquez $C$. La fauna flebotomica (Diptera: psychodidae) de tres provincias de la Región Nor Oriental del Marañon, Perí. Revista da Sociedade Brasileira de Medicina Tropical 28:215-221, jul-set, 1995.

Key-words: Pblebotominae. Perú. Lutzomyia, Brumptomyia, Warileya, bartonellosis. Peruvian Verruga. Carrion's disease.

\section{AGRADECIMIENTOS}

Al profesor Dr. Arístides Herrer por su orientación; asimismo, a los señores: Ricarte Diaz, Raúl Jiménez, Eusebio León, Gilberto Granadino y Lizauro Araujo, quienes apoyaron en los trabajos de campo.

\section{REFERENCIAS BIBLIOGRAFICAS}

1. Alexander JB, Takaoka H, Eshila Y, Gomez EA, Hashiguchi Y. New records of Phlebotomine San flies (Diptera: Psychodidae) from Ecuador. Memórias do Instituto Oswaldo Cruz 87:123-130, 1992.

2. Battistini T. La verruga peruvienne (Sa transmission par le Phlébotome). Revue SudAmericaine de Medicine et de Chirurgie 2:71924, 1931.

3. Cáceres A. Fauna flebotómica (Diptera, Psychodidae) del valle interandino Higueras, Huánuco-Perú. Revista Peruana de Entomología 34:53-54, 1991

4. Cáceres A. Ditribución Geográfica de Lutzomyia verrucarum (Townsend, 1913) (Diptera, Psychodidae, Phlebotominae), vector de la bartonellosis humana en el Perú. Revista do Instituto de Medicina Tropical de São Paulo 35:485-490, 1993.

5. Cáceres A, Canales J, León E, Chang OJ, Van Der Roost D, Llanos-Cuentas A. Estudio entomológico de un área endémica de leishmaniasis tegumentaria en Huancabamba, departamento de Piura (Perú). In: Resumenes de $\mathrm{V}$ Congreso Panamericano de Infetologia, Lima p. II-20, 1991.

6. Cáceres A, Maguiña C, Chauca J. Fauna flebotómica (Diptera: Psychodidae, Phlebotominae) del valle interandino del Santa, Ancash-Perú. Revista Peruana de Entomología 35:7-8, 1992.

7. Cáceres A, Villaseca P, Inga R, López M, Arévalo J, Llanos-Cuentas A. Lutzomyia ayacucbensis (Diptera: Psychodidae, Phlebotominae) probable vector de la leishmaniasis cutánea andina (uta) en Lucanas y Parinacochas (Ayacucho, Perú). In: Resumenes del $\mathrm{V}$ Congreso Panamericano de Infectologia, Lima p. II-22, 1991.

8. Carvajal HL, Paulson BG, Zerega PF, Loaiza VM, Palacios CHM. Bartonellosis en el Ecuador,
Verruga Peruana, su estudio Histórico, Epidemiológico, Inmunológico, Clínico e Histopatológico. Revista Ecuatoriana de Higiene y Medicina Tropical 31:37-47, 1978.

9. Gamarra CA. Geografía Médica de la Verruga Peruana.Verruga Peruana en la zona fronteriza de Canchis-Chinchipe. Revista de la Sanidad de Policía 24:50-53, 1964.

10. Herrer A. Observaciones sobre la verruga en el departamento de Cajamarca. II. Observaciones entomológicas. Revista de Medicina Experimental 2:354-361, 1943.

11. Herrer A. Estudios sobre la leishmaniasis tegumentaria en el Perú. VI. Relación entre leishmaniasis tegumentaria y Pblebotomus. Revista de Medicina Experimental 8:119-137, 1951.

12. Herrer A. Epidemia de la enfermedad de Carrión en Cajabamba (Cajamarca, Perú), 1980. Revista Peruana de Entomología 32:9-18, 1989.

13. Herrer A. Verruga Peruana en la Quebrada de Huarmaca (Huancabamba, Piura), 1981. Revista Peruana de Entomología 32:19-28, 1989.

14. Herrer A. Epidemiología de la Verruga Peruana, Gonzales-Mugaburu (editor), Lima 1990.

15. Hertig M. Cultivo de la Bartanella bacilliformis de un caso de verruga en el Ecuador. Boletín de la Oficina Sanitaria Panamericana 19:756-758, 1940.

16. Hertig M. Phlebotomus and Carrion's disease. American Journal Tropical Medicine 22 (suppl. 5):1-81, 1942.

17. Galati EAB, Cáceres A. Descricâo de Lutzomyia palliditborax, SP.N. e de Lutzomyia castanea, SP.N. do Peru e análise cladistica das séries do subgénero Helcocyrtomyia Barretto (Diptera, Psychodidae). Revista Brasileira do Entomología 38:471-488, 1994

18. Le Pont F, Barrera C, Cáceres A, Galati EAB, Jarrra $O$, Riofrio AR, Mouchet J, Guderian RH. Leishmaniose en Equateur. 6. Nota epidemilogique et entomologique sur le foyer de leishmaniase de Zumba.Annales de la Société de Médecine Tropicale 74:43-49, 1994.

19. Llanos ZB. Los flebotominos del Perú y su distribución geográfica (Diptera. Psychodidae, Phlebotominae). Revista Peruana de Entomología 24:183-184, 1981.

20. Montalván JA. Un foco de bartonellosis en el Ecuador. Boletín de la Oficina Sanitaria Panamericana 19:154, 1940. 
Cáceres $A G$, Galati $E A B$, Le Pont F, Velásquez C. La fauna flebotomica (Diptera: psychodidae) de tres provincias de la Región Nor Oriental del Marañon, Perí. Revista da Sociedade Brasileira de Medicina Tropical 28:215-221, jul-set, 1995.

21. Noguchi H, Shannon RC, Tilden EB, Tyler JR. Etiology of Oroya fever. XIV.The insect vectors of Carrion's disease. Journal of Experimental Medicine 49:993-1008, 1929.

22. Odriozola E. La maladie de Carrión ou la verruga péruvienne. Carré y Naud, Paris, 1898.

23. Odriozola E. Estado actual de nuestros conocimientos acerca de la enfermedad de Carrión o verruga peruana. La Opinión Nacional, Lima, 1908.

24. Patiño-Camargo L. Un nuevo foco de bartonellosis en América. Nota preliminar. Boletín de la Oficina Sanitaria Panamericana 18:305-313, 1939.

25. Pérez E, Villaseca P, Cáceres A, López M, Zolessi A, Campos M, Guerra H, Llanos-Cuentas A. Leishmania (Viannia) peruviana isolated from the sandfly Lutzomyia peruensis (Diptera, Psychodidae) and a sentinel hamster in the Huyllacayan Valley, Ancash, Peru. Transactions of the Royal Society of Tropical Medicine and Hygiene 85:60, 1991.

26. Rebagliati R. Verruga Peruana (enfermedad de Carrión). Imprenta Torres Aguirre, Lima, 1940.
27. Shannon RC. Entomological investigations in connection with Carrion's disease. American Journal of Hygiene 10:78-111, 1929.

28. Solano L, Quiroz C,Alarcón J, Luna A, Chumbe W. Situación epidemiológica de la bartonelosis humana en San Ignacio, Cajamarca, Perú. Boletín del Instituto de Medicina Tropical "Daniel A. Carrión" Universidad Nacional Mayor de San Marcos, Lima, 2:1-4, 1983.

29. Gómez EAL, Hashiguchi Y. Monthly variation in natural infection of the sandfly Lutzomyia ayacucbensis with Leishmania mexicana in an endemic focus in the Ecuadorian Andes. Annales Tropical Medicine and Parasitology 85:407-411, 1991.

30. Townsend CHT. The possible and probable etiology and transmission of verruga fever. Journal of Economic Entomology 6:211-225, $1913 a$.

31. Townsend CHT. The transmission of verruga by Phlebotomus. Journal American Medical Association 61:1717-1718, 1913e. 